\title{
Effects of the Bragg peak degradation due to lung tissue in proton therapy of lung cancer patients
}

\author{
Kilian-Simon Baumann ${ }^{1,2^{*}}$ (D), Veronika Flatten ${ }^{1,2}$, Uli Weber ${ }^{3}$, Stefan Lautenschläger ${ }^{1,4}$, Fabian Eberle $^{1,4}$, \\ Klemens Zink ${ }^{1,2,5}$ and Rita Engenhart-Cabillic ${ }^{1,4}$
}

\begin{abstract}
Purpose: To quantify the effects of the Bragg peak degradation due to lung tissue on treatment plans of lung cancer patients with spot scanning proton therapy and to give a conservative approximation of these effects.

Methods and materials: Treatment plans of five lung cancer patients (tumors of sizes $2.7-46.4 \mathrm{~cm}^{3}$ at different depths in the lung) were optimized without consideration of the Bragg peak degradation. These treatment plans were recalculated with the Monte Carlo code TOPAS in two scenarios: in a first scenario, the treatment plans were calculated without including the Bragg peak degradation to reproduce the dose distribution predicted by the treatment-planning system (TPS). In a second scenario, the treatment plans were calculated while including the Bragg peak degradation. Subsequently, the plans were compared by means of $D_{\text {mean }}, D_{98 \%}$ and $D_{2 \%}$ in the clinical target volume (CTV) and organs at risk (OAR). Furthermore, isodose lines were investigated and a gamma index analysis was performed.

Results: The Bragg peak degradation leads to a lower dose in the CTV and higher doses in OARs distal to the CTV compared to the prediction from the TPS. The reduction of the mean dose in the CTV was $-5 \%$ at maximum and $-2 \%$ on average. The deeper a tumor was located in the lung and the smaller its volume the bigger was the effect on the CTV. The enhancement of the mean dose in OARs distal to the CTV was negligible for the cases investigated.

Conclusions: Effects of the Bragg peak degradation due to lung tissue were investigated for lung cancer treatment plans in proton therapy. This study confirms that these effects are clinically tolerable to a certain degree in the current clinical context considering the various more critical dose uncertainties due to motion and range uncertainties in proton therapy.
\end{abstract}

Keywords: Proton therapy, Lung modulation, Bragg peak degradation, Treatment planning

\section{Background}

Since proposed for radiation therapy, ion beams are of increasing interest in radiation oncology $[1,2]$. In homogeneous materials the dose profile of heavy charged particles such as protons consists of a low dose plateau at small depths followed by the so-called Bragg peak where most of the dose is deposited. The Bragg peak is

\footnotetext{
* Correspondence: kilian-simon.baumann@staff.uni-marburg.de

${ }^{1}$ University Medical Center Giessen-Marburg, Department of Radiotherapy and Radiooncology, Marburg, Germany

${ }^{2}$ University of Applied Sciences, Institute of Medical Physics and Radiation

Protection, Giessen, Germany

Full list of author information is available at the end of the article
}

followed by a sharp distal fall-off. This finite range and sharp distal fall-off of the dose deposition as well as the comparable low entrance dose lead to a reduction in the integral dose. These advantages of protons in radiation therapy lead to the possibility of a conformal dose distribution in the target while sparing surrounding healthy tissue [3]. Mainly two approaches for the use of proton therapy have evolved [4]: 1) to escalate the dose in the tumor while allowing the same dose to organs at risk (OAR) compared to conventional photon therapy and 2) keeping the target dose constant compared to conventional photon therapy and reducing the dose deposited

(c) The Author(s). 2019 Open Access This article is distributed under the terms of the Creative Commons Attribution 4.0 International License (http://creativecommons.org/licenses/by/4.0/), which permits unrestricted use, distribution, and 
to OARs as much as possible. Both approaches can be considered for the treatment of lung cancer patients with protons. On the one hand, it has been shown in some studies that a dose escalation in the tumor improves local control and survival in non-small cell lung cancer (NSCLC) patients [4]. On the other hand, the sparing of healthy tissue is of interest for tumors in difficult anatomies where the tumor is located near to sensitive structures or even enclosed by OARs. This is often the case for lung cancer patients due to the possible proximity of OARs like the heart, esophagus, trachea, large blood vessels and the spinal cord [4].

For early-stage NSCLC patients the outcomes achieved with proton therapy are similar to those achieved with stereotactic body radiotherapy (SBRT) [4] despite excellent dose distributions and sparing of OARs. This holds especially for small peripheral lesions, since these are mostly located far from critical structures (except for ribs and chest wall) and hence a sparing of OARs is well achievable with SBRT. However, for larger tumors, especially those located near to OARS, proton therapy might be superior to SBRT.

For locally advanced (stage III) lung cancer patients, virtual clinical studies showed that in proton plans it is possible to reduce the dose deposition in normal tissue, especially in the heart, compared to photon plans [3-5]. Additionally, a phase II study with 44 patients showed an enhanced median survival in a combined radiochemotherapy when irradiating with protons compared to photons. The patients treated with protons showed minimal grade 3 toxicities $[4,6]$.

In addition to the debatable benefits of proton therapy compared to SBRT in lung cancers, some difficulties in treating lung cancers with protons arise due to the underlying physics as well as the technical application of the proton beam. One of the main issues is the range of protons that depends on the material in the beam path: in case that the patient's anatomy changes and hence is different during the treatment compared to the treatmentplanning process, the range and hence the dose deposition can be different to that predicted by the TPS. Hence, the outcome of the treatment is vulnerable to changes in the anatomy of the patient as the patient moves, is not optimally positioned or the anatomy of the patient changes between fractions, e.g. due to a shrinkage of the tumor or weight loss [7-10]. Especially the change in the anatomy between fractions causes a need in plan adaption strategies for proton therapy [11].

Another issue is that the range of protons is predicted based on X-ray CT images while the conversion of stopping powers from X-ray CT images is inaccurate $[12,13]$. Furthermore, uncertainties in the dose deposited arise from uncertainties in biological effectiveness models $[7,14]$.
A crucial topic in the proton therapy of lung cancer patients is motion management since interplay effects due to respiratory motion or motion of the heart combined with the precise application of actively scanned proton beams can lead to a severe underdosage of the target volume [15-20].

Another uncertainty in proton therapy of lung cancer patients arises from the characteristics of the lung tissue itself: The heterogeneous structure of the lung tissue leads to a degradation of the Bragg peak and to a wider distal fall-off $[21,22]$. If this degradation is not considered correctly during the treatment planning of lung cancer patients, it might lead to an underdosage of the target volume and an overdosage of normal tissue distal to the target volume [22, 23]. Although this degradation has been described in numerous works [24-29], it cannot be considered in the clinical treatment-planning process and dose calculation on treatment-planning CT images. The reason is that due to the restricted resolution of treatment-planning CTs, the microscopic structure of lung tissue is not resolved sufficiently and a more homogeneous tissue distribution is predicted [26, 30].

Baumann et al. [29] introduced and extensively tested an efficient method to consider the Bragg peak degradation on the base of typically used treatment-planning CT data in Monte Carlo codes by applying a density modulation to voxels associated with the lung. Flatten et al. [31] used this model to estimate the effects of the Bragg peak degradation based on a phantom study where spherical tumors of different sizes were placed at different depths in the lung and the underdosage of the target volume was quantified. The result showed that the underdosage of the target volume increases with an increasing depth of the tumor in lung and a decreasing tumor volume. The maximum underdosage in terms of the mean dose was $-15 \%$ compared to the dose distribution predicted by the treatment-planning system that did not consider the Bragg peak degradation.

In this study the effects of the Bragg peak degradation were investigated on clinical cases for various anatomical locations of the tumor in the lung and different treatment plans. We chose simple field configurations so that the results can be used by a large variety of proton centers. The goal is to give upwards estimations for the dose uncertainty in the target volume and OARs. For that, we included also extreme cases (e.g small tumor volumes and large depths in lung) to quantify the maximum degradation effect in realistic patient anatomies.

\section{Methods and materials Selection of patients}

We investigated five exemplary clinical cases with tumor volumes between $2.7 \mathrm{~cm}^{3}$ and $46.4 \mathrm{~cm}^{3}$. The tumors were located in the right lung in the upper lobe (two 
cases) or the central lobe (three cases). We chose the clinical cases to have tumors located in the center of the lung as well as tumors that are located near to soft tissue or OARs. In doing so we are able to investigate different depth of the tumor in the lung as well as the effects of the Bragg peak degradation on surrounding normal tissue and OARs. For two cases the tumor was located near to the spinal cord. No tumor was located directly at the thorax wall in order to always have lung tissue between the thorax wall and the tumor and hence in the beam path. The patients were originally treated with photons and retrospectively re-planned with protons for this study. We used different beam directions in the proton plans to generate different path lengths in the lung (see Fig. 1 and Table 1).

As described in the introduction, small tumor volumes as investigated in this study might not benefit from proton therapy compared to SBRT and hence tend to be less relevant for proton therapy. Yet, small tumor volumes have been treated with protons at different centers [32-34], with volumes going down to only $1 \mathrm{~cm}^{3}$. Furthermore, Flatten et al. [31] showed that the effects of the Bragg peak degradation increase with a decreasing tumor volume. Thus, we investigated these small tumors as well, in particular to derive an upwards estimation for larger and thus clinically more relevant tumor volumes.

\section{Treatment planning}

All treatment plans were optimized with Eclipse v.13.7 (VARIAN) using the non-linear universal proton optimizer, v.13.7.15. The total prescribed dose was $30 \mathrm{~Gy}$ (RBE) and the only planning objective was to deliver at least $95 \%$ of the prescription dose to at least $98 \%$ of the planning target volume (PTV). For small tumor volumes we accepted hot spots (up to $115 \%$ of the prescribed dose) in the PTV. The PTV was the clinical target volume (CTV) plus an isotropic margin of $3 \mathrm{~mm}$, although most PTV concepts proposed in the literature [7, 34-36] are field specific and account for uncertainties of the proton's range or the positioning of the patient. However, in the majority of clinical situations, an isotropic margin around the CTV is used for treatment planning as done in this study. Additionally, we planned on static CT data, ignoring movements of the anatomy due to respiration in both the planning and dose calculation. By excluding any effects due to motion, positioning of the patient or range uncertainties, we are able to extract the pure effects of the Bragg peak degradation. We analyzed these effects on the CTV and not the PTV, since the CTV

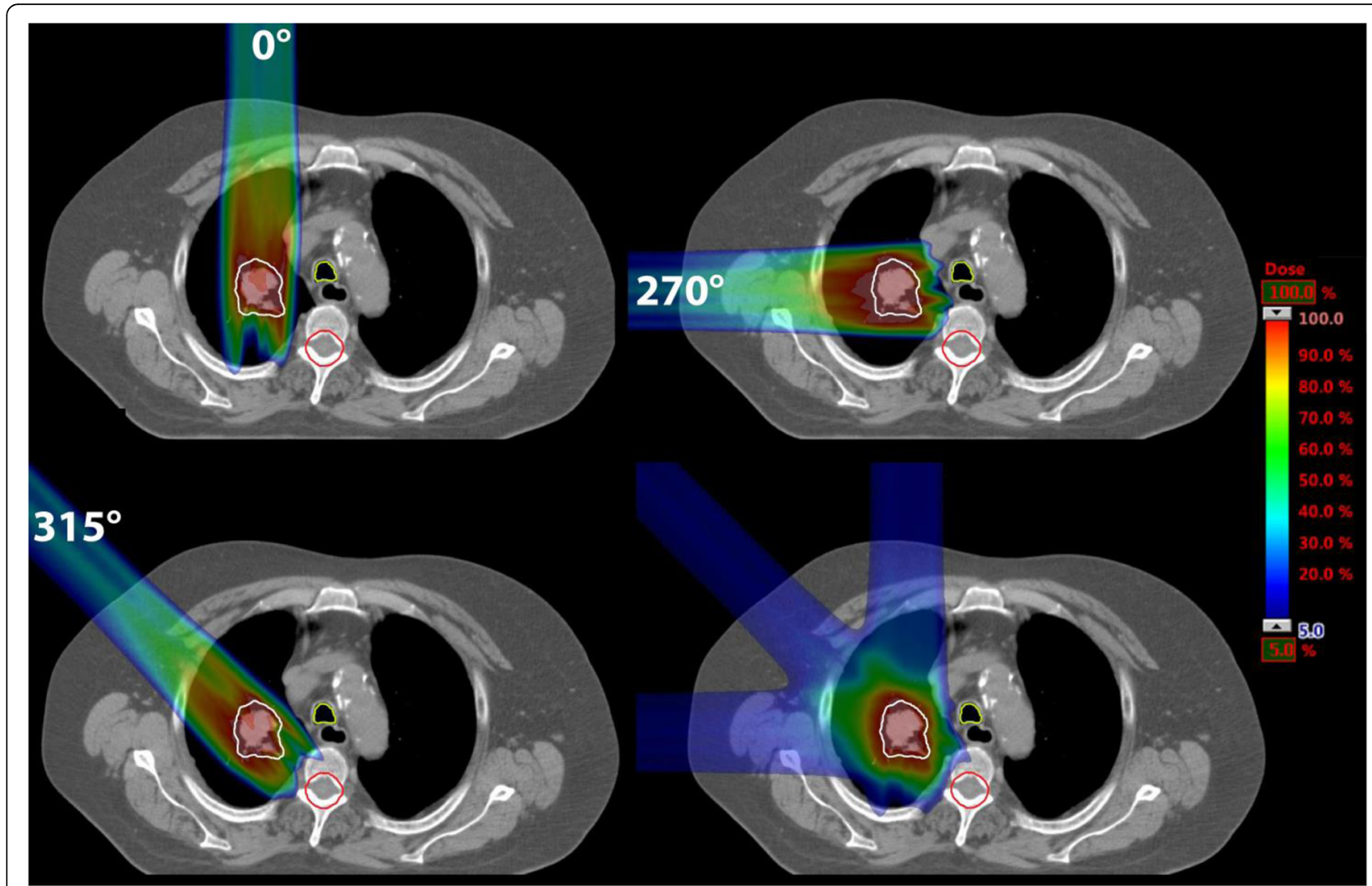

Fig. 1 CT slices of one exemplary patient (patient 1): for the beam directions $0^{\circ}, 270^{\circ}$ and $315^{\circ}$ plans were optimized individually consisting of one single field. On the bottom right the sum of these plans is shown. The CTV is marked in white, trachea in light green and spinal cord in red. On the right a color bar is given indicating the relative dose 
Table 1 Volumes of the CTVs and lungs for the five patients and minimum and maximum diameter of the CTVs as well as depths in lung of the CTVs for each beam direction. The depth of the CTV corresponds to the path length through lung tissue for the spot at the isocenter

\begin{tabular}{|c|c|c|c|c|c|c|}
\hline \multirow[t]{2}{*}{ Patient } & \multirow{2}{*}{$\begin{array}{l}\text { Lung volume } \\
\text { in } \mathrm{cm}^{3}\end{array}$} & \multirow{2}{*}{$\begin{array}{l}\text { Volume of } \\
\text { CTV in } \mathrm{cm}^{3}\end{array}$} & \multirow{2}{*}{$\begin{array}{l}\min . / \max . \\
\text { diameter of } \\
\text { CTV in } \mathrm{cm}\end{array}$} & \multicolumn{3}{|c|}{ Depth of CTV in cm for different beam directions } \\
\hline & & & & $0^{\circ}$ & $270^{\circ}$ & $315^{\circ}$ \\
\hline 1 & 2294 & 46.4 & $2.9 / 4.2$ & 6.2 & 3.3 & 3.6 \\
\hline 2 & 1882 & 4.2 & $1.8 / 2.3$ & 1.8 & 2.1 & 1.5 \\
\hline 3 & 1705 & 32.1 & $3.5 / 5.2$ & 12.2 & 9.2 & 9.2 \\
\hline 4 & 1780 & 6.2 & $2.2 / 2.4$ & 6.9 & 2.7 & 5.3 \\
\hline 5 & 1600 & 2.7 & $1.6 / 2.0$ & 4.5 & 3.8 & 3.6 \\
\hline
\end{tabular}

is the clinically relevant structure. Since for each optimized plan the dose deposited in the OARs was below any critical value we did not include any constraints for OARs in the treatment-plan optimization.

We decided to investigate simple treatment plans consisting of only one single field coming from either $0^{\circ}, 270^{\circ}$ or $315^{\circ}$. We used different beam directions to generate different depths of the CTV in the lung. The distal spot spacing was $3 \mathrm{~mm}$, the lateral spot spacing was 0.45 times the full-width-half-maximum (FWHM) in air. The FWHM for $70 \mathrm{MeV}$ protons was $32.5 \mathrm{~mm}$ and for $221 \mathrm{MeV}$ protons it was $8.1 \mathrm{~mm}$.

The different beam directions are shown in Fig. 1 for one exemplary patient. As described later in the text, we also made a sum plan of the three single field plans for each patient as shown in Fig. 1 bottom right. In Fig. 2 the remaining four patients are shown with one exemplary treatment plan each.

Although some of these beams may not be the best choice from a clinical point of view (e.g. OARs distal to the PTV, large depth of the tumor in the lung), we decided to investigate these cases anyway to give an upwards estimation for the effects of the Bragg peak degradation also for worst-case scenarios.

The motivation for using simple treatment plans is to highlight the effects of the Bragg peak degradation. Furthermore, there is no gold standard in plan design for lung cancer patients, especially concerning the choice of number of fields and beam directions, although several proton centers have already treated lung cancer patients with protons [6, 32-34]. Thus, in keeping the treatment plans simple, we can assure that the results from this study are usable for as many different proton centers as possible since the dependencies of the Bragg peak degradation (e.g. on the depth of tumor in lung) can be assessed more easily using simple treatment plans compared to complex IMPT plans.

In order to assess whether the results from this study can be used to estimate the effects of the Bragg peak degradation for more complex plans, we investigated two IMPT plans, one each for patient 1 and 5 as shown in Fig. 3. The choice fell on these two patients since they have the largest and smallest tumor volume (compare Table 1). Multi-field optimization was enabled to optimize three fields for each plan. The same PTV concept and planning objectives were used as for the simple plans. For patient 1 the beam directions were $180^{\circ}, 270^{\circ}$ and $330^{\circ}$. For patient 5 the beam directions $10^{\circ}, 180^{\circ}$ and $270^{\circ}$ were used.

\section{Simulations}

Simulations were performed using the Monte Carlo code TOPAS (Tool for Particle Simulations) version 3.1.p1 [37], a toolkit based on Geant4 (Geometry And Tracking) version geant4-10-03-patch-01 [38]. We used the same beam data in both TOPAS and Eclipse and commissioned these data to match the beam delivery system at the Ion-Beam Therapy Center Marburg. Dose calculation results in water between TOPAS and Eclipse agreed well. The passing rate of the gamma index $1 \% / 1 \mathrm{~mm}$ for voxels with dose values greater than $20 \%$ of the maximum dose was larger than $98 \%$ for single spots. It is known that differences between dose calculation algorithms as used in Eclipse and Monte Carlo codes such as TOPAS exist especially for dose calculations in the lung [39]. Hence, all dose calculations were performed with TOPAS so that differences in the dose calculation between TOPAS and Eclipse do not falsify the results.

Each treatment plan optimized with Eclipse was recalculated in TOPAS in two scenarios: In the first scenario, each optimized treatment plan was calculated on the original CT data. Hence, this calculation corresponds to the prediction from the treatment-planning system. In a second scenario, the plans were recalculated while considering the Bragg Peak degradation. To do so, we used the mathematical model presented by Baumann et al. [29]. The strength of the Bragg peak degradation is quantified by the material characteristic "modulation power" $P_{\text {mod }}$ : The greater the modulation power of a heterogeneous material like lung tissue the broader the 


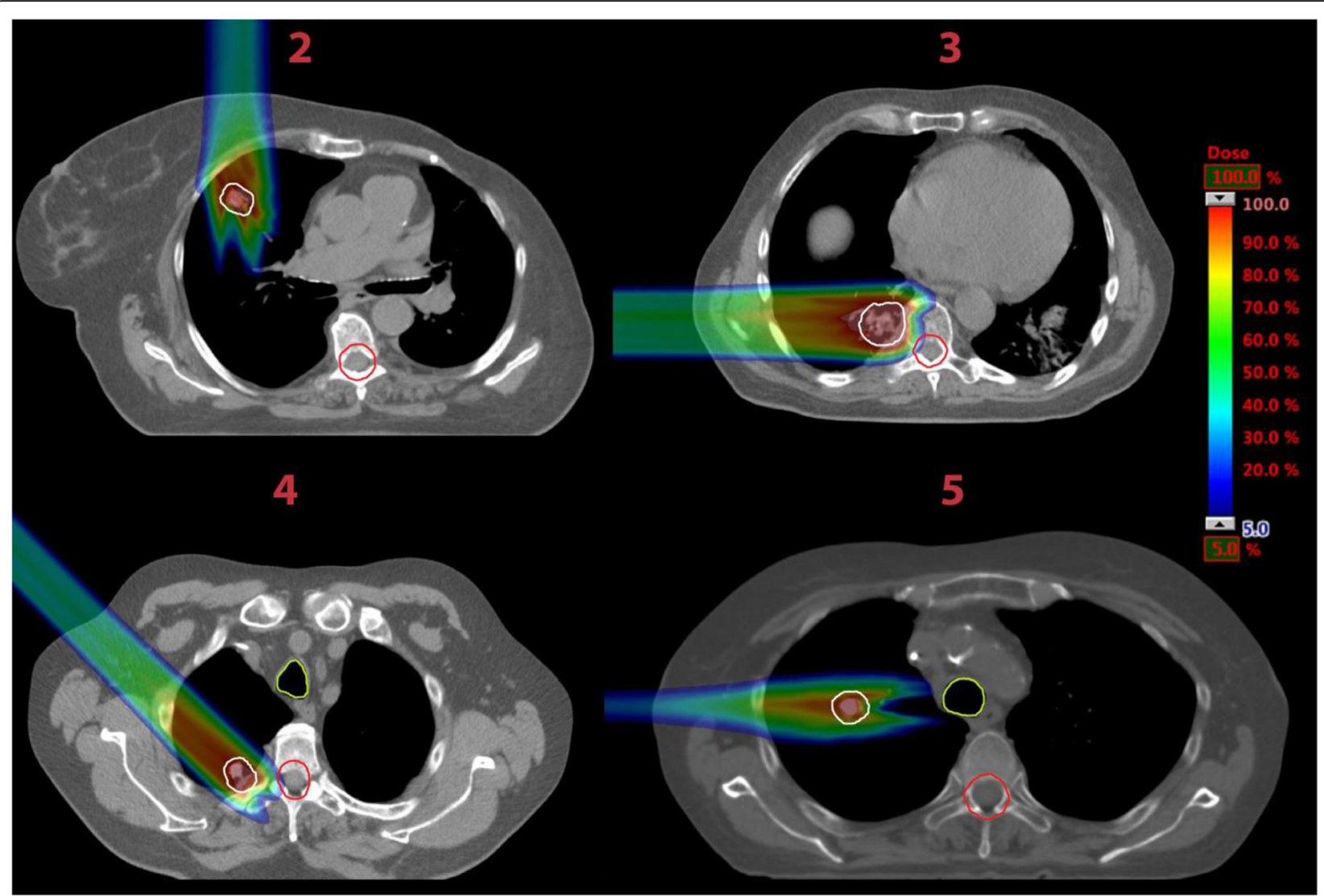

Fig. 2 CT slices of the remaining patients. The patient's numbers are marked in red. For each patient one exemplary plan is shown. The CTV is marked in white, trachea in light green and spinal cord in red. On the right a color bar is given indicating the relative dose

Bragg peak and the less steep the distal fall-off as shown in Fig. 4. Based on the modulation power a density distribution can be derived. When modulating the density of each voxel associated with the lung within the patient following this density distribution, the Bragg peak degradation due to the lung tissue is being reproduced [29]. The dose distributions for each plan obtained from using the original CT data (non-modulated case) and when applying the density modulation (modulated case) were compared in means of cumulative dose volume histograms (DVH), mean doses $\mathrm{D}_{\text {mean }}, \mathrm{D}_{98 \%}$ and $\mathrm{D}_{2 \%}$ in the CTV and OARs. Additionally, we investigated the differences in the dose distribution when combining the dose distributions from the single plans (beam directions $0^{\circ}$,

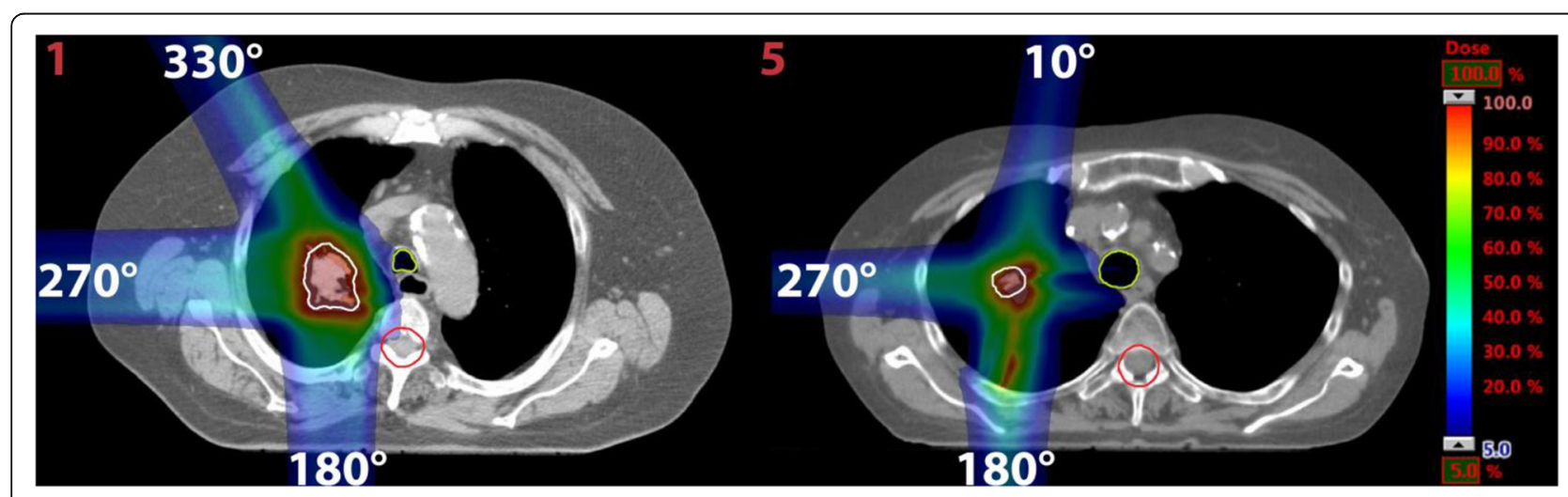

Fig. 3 CT slices of the patients 1 and 5 (marked in red numbers) with the dose distributions for the optimized IMPT plans. The CTV is marked in white, trachea in light green and spinal cord in red. On the right a color bar is given indicating the relative dose 


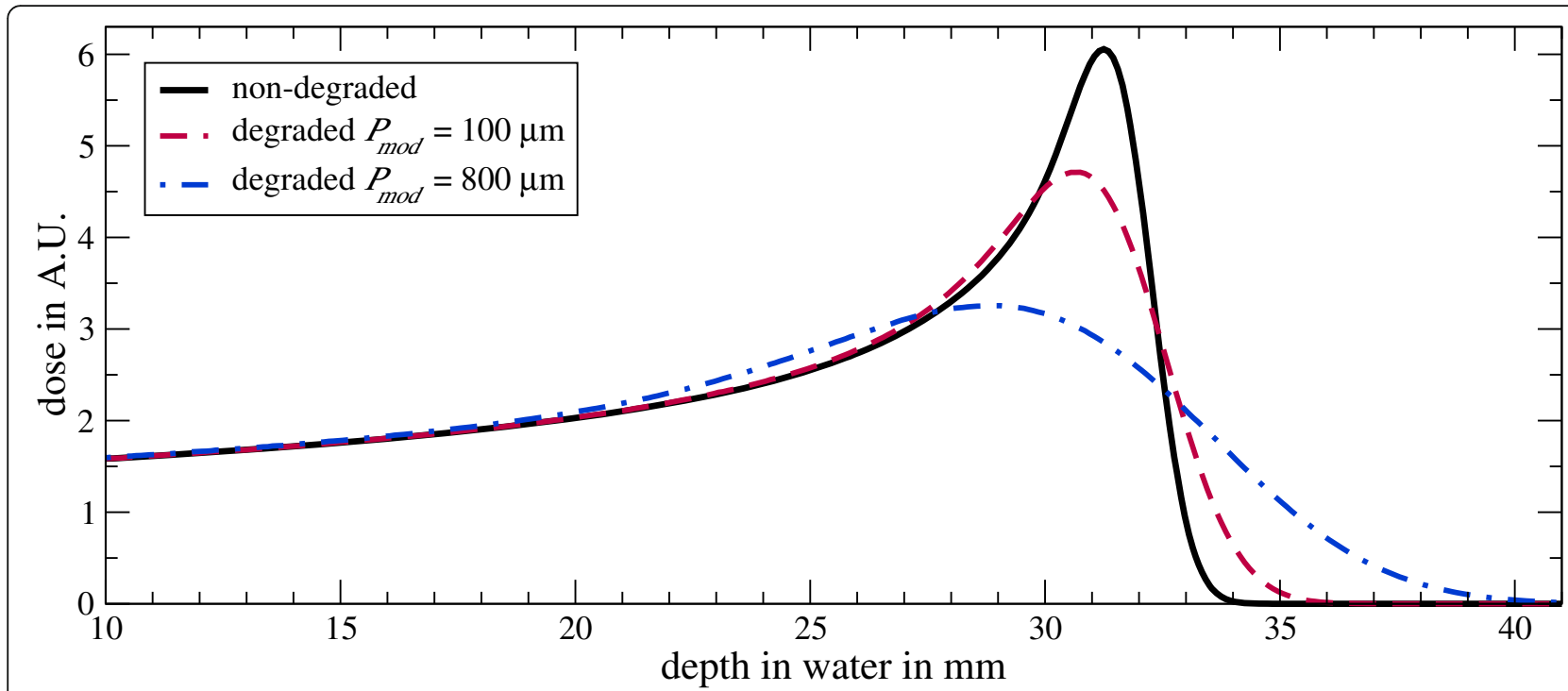

Fig. 4 Depth dose curves of protons in water. In black the Bragg curve as predicted by the treatment-planning system that does not consider the Bragg peak degradation due to the lung tissue. In red and blue the degraded Bragg curves based on modulation powers $P_{\bmod }$ of $100 \mu \mathrm{m}$ and $800 \mu \mathrm{m}$. The greater the modulation power the broader the Bragg peak and the less steep the distal fall-off

$270^{\circ}$ and $315^{\circ}$ ) for each patient to an added-up dose distribution to investigate the influence of the number of irradiated fields on the degradation effects.

In a study by Witt [40] the modulation powers of porcine lungs were measured in proton beams to be in the range from $300 \mu \mathrm{m}$ to $750 \mu \mathrm{m}$. Since the measurements were performed with complete lungs, the measured modulation powers correspond to integrated modulation powers of all the structures of the lung being arranged in the beam that cannot be identified in CT images. The modulation power increases with increasing structure sizes [29]. Thus, the modulation power varies with the position in the lung. In the peripheral region of the lung the modulation power tends to be smaller compared to the central lung where the size of the structures is greater. For almost each measurement the modulation power was in the range from $300 \mu \mathrm{m}$ to $500 \mu \mathrm{m}$ with an average of $450 \mu \mathrm{m}$. For one measurement the modulation power was $750 \mu \mathrm{m}$. However, for this measurement the lung was positioned in a way that a large bronchial structure was in the beam line.

To clarify whether these results obtained with porcine lungs are applicable for human lung tissue, high-resolution CT images with a resolution of $4 \mu \mathrm{m}$ of human lung tissue samples were investigated by Witt [40] and Baumann et al. [29]. The so-gained modulation powers were in the range from $50 \mu \mathrm{m}$ to $250 \mu \mathrm{m}$. The authors discussed that the preparation of the tissue samples resulted in a noticeable loss of water of up to $37 \%$ and hence in a reduction of the sizes of the lung structures. Therefore, the modulation powers gained in this investigation are lower compared to the measurements with porcine lungs.
Both the measurements with porcine lungs and the investigation of human lung tissue samples indicate that the modulation power of lung tissue is in the order of some hundred micrometers. However, until now there is no possibility to determine a patient-specific modulation power for each region of the lung. Therefore, in this study we investigated the effects of the Bragg peak degradation based on modulation powers of $100 \mu \mathrm{m}$, $250 \mu \mathrm{m}, 450 \mu \mathrm{m}$ and $800 \mu \mathrm{m}$, covering the whole range of modulation powers found in the measurements of porcine lungs and the investigation of human lung tissue samples with some additional buffer to determine the minimum and maximum degradation effects in exemplary clinical cases. For the IMPT plans we only used a modulation power of $450 \mu \mathrm{m}$.

\section{Results}

In Fig. 5 on the left side the DVH for patient 1 and the beam direction $270^{\circ}$ is shown for the CTV and the OAR trachea. The Monte Carlo calculated DVH for each volume is shown for the non-modulated case representing the dose distribution predicted by the treatment-planning system and the modulated cases where the Bragg peak degradation based on modulation powers between $100 \mu \mathrm{m}$ and $800 \mu \mathrm{m}$ is considered. The dose coverage of the CTV decreases with an increasing modulation power. The dose deposited in the trachea increases with an increasing modulation power. On the right side the depth dose curves along the center of the beam for the non-modulated scenario and the modulated one based on the maximum modulation power of $800 \mu \mathrm{m}$ are shown. The positions of the body, the lung and the CTV are marked by dashed black lines. We 

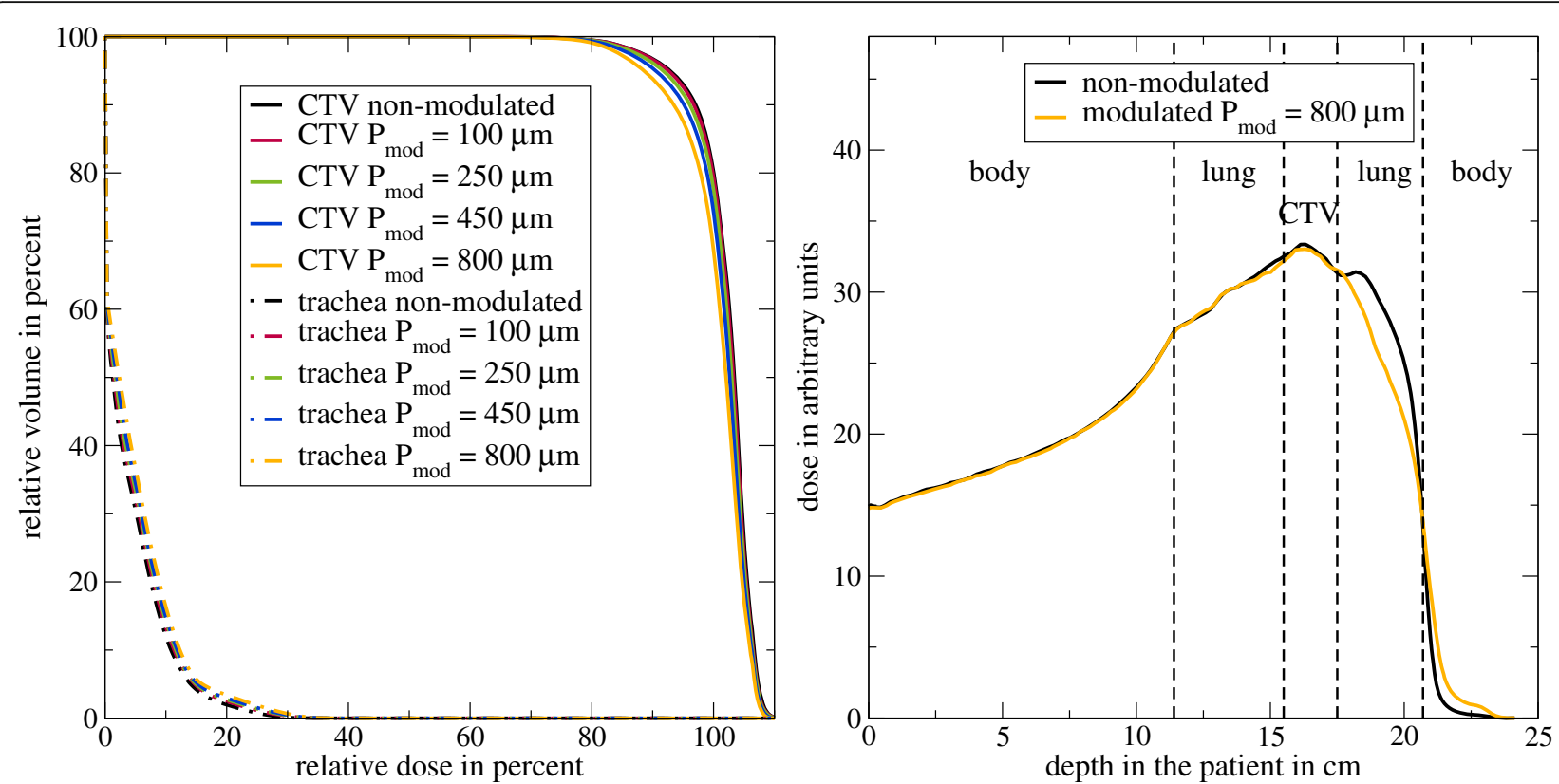

Fig. 5 Left side: Cumulative dose-volume histogram for the CTV and trachea for patient 1 and the beam direction $270^{\circ}$ for both the nonmodulated case and the modulated cases based on modulation powers between $100 \mu \mathrm{m}$ and $800 \mu \mathrm{m}$. Right side: depth dose curve along the center of the beam. The positions of the body, the lung and the CTV are marked by dashed black lines

decided to show the results for the extreme modulation power of $800 \mu \mathrm{m}$ since for a smaller modulation power the effects are not visible as clearly: when entering the body both dose distributions are the same. In the lung the effects of the degradation can be seen resulting in a broader dose curve and a less steep fall-off for the modulated case. Within the CTV there is a slightly higher dose for the nonmodulated case. In the lung distal to the CTV there is a significantly higher dose for the non-modulated case. The background is that in this case a spot is used by the TPS where the Bragg peak is located distal to the CTV in order to achieve a sufficient dose coverage within the CTV. This peak is smoothed in the modulated case as described by Flatten et al. [31]. In the body distal to the lung the dose for the modulated case is higher due to the broader fall-off resulting in a larger range and hence a higher dose deposition.

In Fig. 6 exemplary isodose lines [41, 42] for 95, 80 and $20 \%$ of the prescribed dose are shown for the nonmodulated case (pink) and the modulated case based on a modulation power of $800 \mu \mathrm{m}$ (green). Additionally, the CTV is marked in white, the trachea in light green and the spinal cord in red. Again, we decided to show the results for the extreme modulation power of $800 \mu \mathrm{m}$. The $95 \%$ isodose lines are shown in the left column, the $80 \%$ in the middle column and the $20 \%$ in the right column. In the first line the isodose lines for patient 1 (marked with a white number) are shown for the beam direction $270^{\circ}$. The regions enclosed by the 95 and $80 \%$ isodose lines are larger for the non-modulated cases indicating the underdosage of the CTV due to the Bragg peak degradation (compare DVH and depth dose in Fig. 5). The $20 \%$ isodose line for the modulated case reaches farther compared to the non-modulated case. The same effects can be seen for all patients.

The greater range of the $20 \%$ isodose lines shows the potential risk of an overdosage in OARs distal to the target volume. Especially for patient 3 and 4 in Fig. 6 the isodose lines for $20 \%$ of the prescribed dose reach into the spinal cord. However, the additional range of the $20 \%$ isodose lines is $2 \mathrm{~mm}$ at maximum for these two patients. The maximum shift of each isodose line for the modulated case compared to the non-modulated case for the patients as shown in Fig. 6 are listed in Table 2 . A negative sign marks a shorter range compared to the non-modulated case. The range uncertainties of the isodose lines in lung (patient 1 and patient 2 except for the $20 \%$ isodose line) are larger compared to those in tissue (patient 3 and 4 all isodose lines and 20\% isodose line of patient 1).

To quantify the effects shown in Figs. 5 and 6 in terms of dose, the absolute dose values for the non-modulated case and the differences in percent of the mean dose $D_{\text {mean }}, D_{98 \%}$ (the dose that is received by $98 \%$ percent of the volume) and $D_{2 \%}$ between the modulated cases and the non-modulated case for patient 1 are reported in Table 3 for the CTV, the trachea and the spinal cord. The $\mathrm{D}_{98 \%}$ is taken to quantify the minimum dose received by a volume while the $\mathrm{D}_{2 \%}$ is taken to quantify the maximum dose received by a volume. For the OARs 

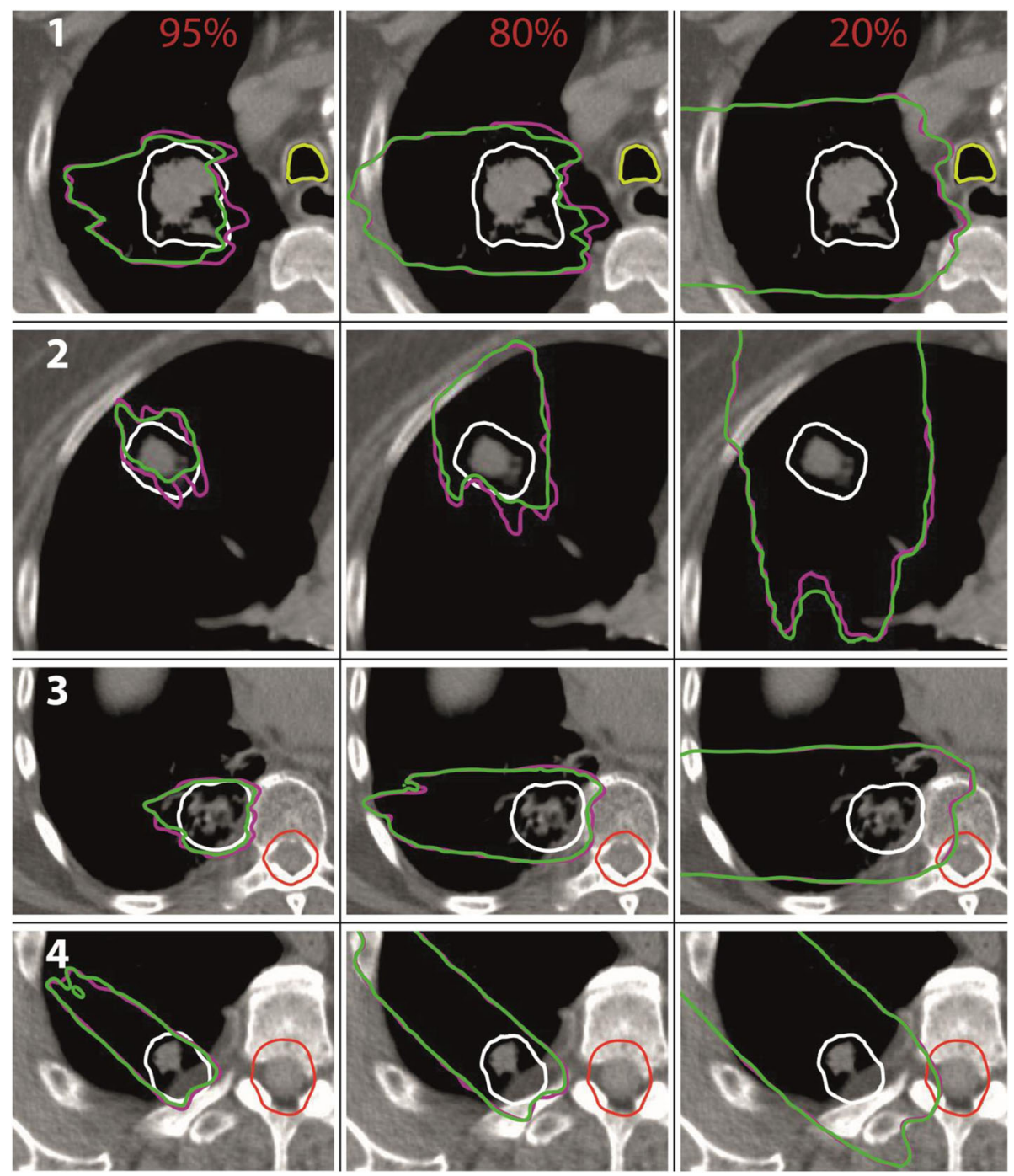

Fig. 6 Isodose lines for 95, 80 and 20\% of the prescribed dose. In pink for the non-modulated case, in green for the modulated case based on a modulation power of $800 \mu \mathrm{m}$. In the first column the $95 \%$, in the middle column the $80 \%$ and in the right column the $20 \%$ isodose lines. Different patient cases are marked in white numbers. The CTV is marked in white, the trachea in light green and the spinal cord in red

the mean dose $\mathrm{D}_{\text {mean }}$ and the $\mathrm{D}_{2 \%}$ as a quantification of the maximum dose are shown.

The mean dose $\mathrm{D}_{\text {mean }}, \mathrm{D}_{98 \%}$ and $\mathrm{D}_{2 \%}$ in the CTV are smaller for the modulated cases compared to the nonmodulated case and hence the prediction from the treatment-planning system. The differences increase with an increasing modulation power. The largest differences can be seen for the beam direction $0^{\circ}$ (corresponding to the largest depth in lung) and the maximum modulation power of $800 \mu \mathrm{m}$. Concerning the OARs trachea and spinal cord, the doses deposited in the modulated cases are greater compared to the nonmodulated case for the beam directions $270^{\circ}$ and $315^{\circ}$. The differences increase with an increasing modulation power. The maximum difference in the mean dose $D_{\text {mean }}$ is $+24 \%$ for the trachea as well as the spinal cord.
However, these relative deviations correspond to low absolute deviations of $0.3 \mathrm{~Gy}$ and $<0.1 \mathrm{~Gy}$, respectively. The largest difference for the $\mathrm{D}_{2 \%}$ value for the trachea is $+24 \%$ and $+21 \%$ for the spinal cord. These deviations correspond to $1.5 \mathrm{~Gy}$ and $0.2 \mathrm{~Gy}$, respectively. It can be seen, that the effects of the Bragg peak degradation on the OARs are almost non-existent for a beam direction $0^{\circ}$ since in this case no OAR is positioned distal to the PTV. The effects on the trachea are largest for the beam direction $270^{\circ}$. For the spinal cord the effects are largest for the beam direction $315^{\circ}$.

In Table 4 the absolute dose values and the differences in percent of the mean dose $D_{\text {mean }}, D_{98 \%}$ and $D_{2 \%}$ for the CTV between the modulated cases and the nonmodulated case are shown for the patients 2 to 5 . The results are given for a modulation power of $800 \mu \mathrm{m}$ to 
Table 2 Maximum shift in $\mathrm{mm}$ of the isodose lines for the modulated case based on a modulation power of $800 \mu \mathrm{m}$ compared to the non-modulated case for the patients and beam directions as shown in Fig. 6. A negative sign stands for a shorter range

\begin{tabular}{llll}
\hline $\begin{array}{l}\text { Patient/beam } \\
\text { direction }\end{array}$ & \multicolumn{4}{l}{ Maximum range uncertainty in $\mathrm{mm}$ for isodose lines } \\
\cline { 2 - 4 } & $95 \%$ & $80 \%$ & $20 \%$ \\
\hline $1 / 270^{\circ}$ & -8 & -5 & 3 \\
$2 / 0^{\circ}$ & -8 & -10 & 5 \\
$3 / 270^{\circ}$ & -4 & -3 & 2 \\
$4 / 315^{\circ}$ & -2 & -2 & 2 \\
\hline
\end{tabular}

give an estimation of the maximum effects. Additionally, the results for a more realistic modulation power of $450 \mu \mathrm{m}$ are given. The deviations between the modulated cases and the non-modulated case for the OARs are as small and negligible as for patient 1 and hence not shown for the other patients. As it is the case with patient 1 , the dose coverage of the CTV in the modulated cases is lower compared to the non-modulated case.

For a modulation power of $800 \mu \mathrm{m}$ the maximum differences in the mean dose $D_{\text {mean }}$ as well as the $D_{98 \%}$ are roughly $-5 \%$. For the $D_{2}$ it is about $-4 \%$. The average difference in the mean dose $D_{\text {mean }}$ is in the order of $-2 \%$, for $\mathrm{D}_{98 \%}$ it is $-3 \%$ and for $\mathrm{D}_{2 \%}$ it is about $-2 \%$.

For a more realistic modulation power of $450 \mu \mathrm{m}$ the maximum differences in the mean dose $\mathrm{D}_{\text {mean }}$, the $\mathrm{D}_{98 \%}$ as well as the $D_{2 \%}$ are roughly $-3 \%$. The average difference in the mean dose $D_{\text {mean }}$ is in the order of $-1 \%$, for $D_{98 \%}$ it is roughly $-2 \%$ and for $D_{2 \%}$ it is $-1 \%$.

Additionally, we looked at the differences in the mean dose $D_{\text {mean }}, D_{98 \%}$ and $D_{2 \%}$ for the CTV between the modulated cases and the non-modulated case when all three plans from the beam directions $0^{\circ}, 270^{\circ}$ and $315^{\circ}$ are combined. As for the irradiation with one single field, in the combined scenario with three fields, the differences between the modulated cases and the nonmodulated case are at maximum about $-5 \%$ for a modulation power of $800 \mu \mathrm{m}$. For a modulation power of $450 \mu \mathrm{m}$ the maximum difference is about $-3 \%$.

In Table 5 the passing rates for the gamma index $3 \% / 1 \mathrm{~mm}$ (local) are shown for each patient and the dose distributions based on modulation powers of $450 \mu \mathrm{m}$ and $800 \mu \mathrm{m}$. All voxels with at least $20 \%$ of the maximum dose were included in the analysis. We chose to set a small

Table 3 Absolute dose values for the non-modulated case and differences in percent of the mean dose $D_{\text {mean, }} D_{98 \%}$ (only for the CTV) and $D_{2} \%$ for the CTV and OARs between the modulated and the non-modulated cases for patient 1

\begin{tabular}{|c|c|c|c|c|c|c|}
\hline \multirow[t]{2}{*}{ Modulation } & \multicolumn{3}{|l|}{ CTV } & \multicolumn{2}{|c|}{ Trachea } & Spinal cord \\
\hline & $D_{\text {mean }}$ & $D_{98 \%}$ & $D_{2 \%}$ & $D_{\text {mean }}$ & $D_{2 \%}$ & $\mathrm{D}_{\text {mean }}$ \\
\hline
\end{tabular}

beam direction: $0^{\circ}$ (depth in lung: $6.2 \mathrm{~cm}$ )

$\begin{array}{llllllll}\text { D }_{\text {non-mod }} \text { in Gy (RBE) } & 29.9 & 25.7 & 32.2 & 0.1 & 0.5 & <0.1 & 0.1 \\ 100 \mu \mathrm{m} & -0.3 \% & -0.4 \% & -0.4 \% & -1 \% & +1 \% & 0 \% & -1 \% \\ 200 \mu \mathrm{m} & -0.7 \% & -1.2 \% & -0.8 \% & 0 \% & +1 \% & 0 \% & -1 \% \\ 450 \mu \mathrm{m} & -1.3 \% & -3.0 \% & -1.2 \% & 0 \% & +2 \% & 0 \% & -1 \% \\ 800 \mu \mathrm{m} & -2.1 \% & -4.9 \% & -1.8 \% & 0 \% & +2 \% & +1 \% & -1 \%\end{array}$

beam direction: $270^{\circ}$ (depth in lung: $3.3 \mathrm{~cm}$ )

\begin{tabular}{|c|c|c|c|c|c|c|c|}
\hline $\mathrm{D}_{\text {non-mod }}$ in Gy (RBE) & 30.1 & 27.3 & 30.4 & 1.1 & 5.6 & 0.1 & 0.7 \\
\hline $100 \mu \mathrm{m}$ & $-0.2 \%$ & $-0.1 \%$ & $-0.2 \%$ & $+7 \%$ & $+9 \%$ & $+2 \%$ & $+6 \%$ \\
\hline $200 \mu \mathrm{m}$ & $-0.5 \%$ & $-1.0 \%$ & $-0.3 \%$ & $+12 \%$ & $+13 \%$ & $+2 \%$ & $+9 \%$ \\
\hline $450 \mu \mathrm{m}$ & $-0.5 \%$ & $-2.2 \%$ & $-0.5 \%$ & $+16 \%$ & $+17 \%$ & $+7 \%$ & $+14 \%$ \\
\hline $800 \mu \mathrm{m}$ & $-0.9 \%$ & $-4.1 \%$ & $-0.6 \%$ & $+24 \%$ & $+24 \%$ & $+9 \%$ & $+21 \%$ \\
\hline
\end{tabular}

beam direction: $315^{\circ}$ (depth in lung: $3.6 \mathrm{~cm}$ )

\begin{tabular}{llllllll}
$D_{\text {non-mod in Gy (RBE) }}$ & 29.8 & 26.6 & 41.6 & 0.2 & 1.0 & 0.1 & 0.7 \\
$100 \mu \mathrm{m}$ & $0.0 \%$ & $0.0 \%$ & $+0.1 \%$ & $+3 \%$ & $+2 \%$ & $+6 \%$ & $+7 \%$ \\
$200 \mu \mathrm{m}$ & $-0.4 \%$ & $-1.5 \%$ & $-0.2 \%$ & $+2 \%$ & $+1 \%$ & $+11 \%$ & $+11 \%$ \\
$450 \mu \mathrm{m}$ & $-0.8 \%$ & $-1.5 \%$ & $-0.4 \%$ & $+2 \%$ & $+2 \%$ & $+15 \%$ & $+14 \%$ \\
$800 \mu \mathrm{m}$ & $-1.5 \%$ & $-3.0 \%$ & $-0.6 \%$ & $+5 \%$ & $+5 \%$ & $+24 \%$ & $+21 \%$ \\
\hline
\end{tabular}


Table 4 Absolute dose values for the non-modulated case and differences in percent of the mean dose $D_{\text {mean, }}, D_{98 \%}$ and $D_{2 \%}$ between the modulated and the non-modulated cases for the CTV and the patients 2 to 5. The modulation powers used in these cases are $450 \mu \mathrm{m}$ and $800 \mu \mathrm{m}$

\begin{tabular}{|c|c|c|c|c|c|c|c|c|c|c|}
\hline \multirow[t]{2}{*}{ Patient } & \multirow[t]{2}{*}{ Modulation } & \multicolumn{3}{|c|}{ Beam direction: $0^{\circ}$} & \multicolumn{3}{|c|}{ Beam direction: $270^{\circ}$} & \multicolumn{3}{|c|}{ Beam direction: $315^{\circ}$} \\
\hline & & $D_{\text {mean }}$ & $D_{98 \%}$ & $\mathrm{D}_{2 \%}$ & $D_{\text {mean }}$ & $D_{98 \%}$ & $\mathrm{D}_{2 \%}$ & $D_{\text {mean }}$ & $D_{98 \%}$ & $\mathrm{D}_{2 \%}$ \\
\hline \multirow[t]{3}{*}{2} & $D_{\text {non-mod }}$ in Gy (RBE) & 29.9 & 23.4 & 32.0 & 30.1 & 25.2 & 31.1 & 30.0 & 22.1 & 32.3 \\
\hline & $450 \mu \mathrm{m}$ & $-1.8 \%$ & $-0.7 \%$ & $-0.2 \%$ & $-1.1 \%$ & $-0.8 \%$ & $-0.7 \%$ & $-1.1 \%$ & $-1.5 \%$ & $-0.7 \%$ \\
\hline & $800 \mu \mathrm{m}$ & $-3.1 \%$ & $-2.9 \%$ & $-3.8 \%$ & $-1.9 \%$ & $-1.4 \%$ & $-1.1 \%$ & $-2.0 \%$ & $-2.5 \%$ & $-1.2 \%$ \\
\hline \multirow[t]{3}{*}{3} & $\mathrm{D}_{\text {non-mod }}$ in Gy (RBE) & 30.0 & 25.8 & 32.2 & 30.1 & 28.3 & 31.5 & 30.0 & 27.0 & 31.9 \\
\hline & $450 \mu \mathrm{m}$ & $-1.1 \%$ & $-1.4 \%$ & $-0.9 \%$ & $-0.6 \%$ & $-2.9 \%$ & $-0.5 \%$ & $-0.8 \%$ & $-2.2 \%$ & $-0.8 \%$ \\
\hline & $800 \mu \mathrm{m}$ & $-1.8 \%$ & $-2.8 \%$ & $-1.5 \%$ & $-1.1 \%$ & $-5.1 \%$ & $-0.8 \%$ & $-1.4 \%$ & $-4.2 \%$ & $-1.1 \%$ \\
\hline \multirow[t]{3}{*}{4} & $\mathrm{D}_{\text {non-mod }}$ in Gy (RBE) & 30.0 & 25.7 & 31.0 & 30.0 & 24.2 & 32.2 & 30.0 & 26.8 & 30.8 \\
\hline & $450 \mu \mathrm{m}$ & $-1.3 \%$ & $-2.5 \%$ & $-0.8 \%$ & $-0.6 \%$ & $-1.2 \%$ & $-0.3 \%$ & $-0.6 \%$ & $-1.1 \%$ & $-0.4 \%$ \\
\hline & $800 \mu \mathrm{m}$ & $-2.2 \%$ & $-4.2 \%$ & $-1.2 \%$ & $-1.0 \%$ & $-2.0 \%$ & $-0.5 \%$ & $-1.0 \%$ & $-2.0 \%$ & $-0.7 \%$ \\
\hline \multirow[t]{3}{*}{5} & $D_{\text {non-mod }}$ in Gy (RBE) & 30.1 & 27.2 & 32.8 & 30.0 & 23.7 & 31.6 & 30.1 & 27.9 & 33.8 \\
\hline & $450 \mu \mathrm{m}$ & $-2.6 \%$ & $-0.5 \%$ & $-1.1 \%$ & $-2.0 \%$ & $-3.0 \%$ & $-2.1 \%$ & $-3.0 \%$ & $-1.5 \%$ & $-3.2 \%$ \\
\hline & $800 \mu \mathrm{m}$ & $-4.7 \%$ & $-2.8 \%$ & $-2.1 \%$ & $-3.1 \%$ & $-4.6 \%$ & $-3.2 \%$ & $-4.9 \%$ & $-2.5 \%$ & $-4.3 \%$ \\
\hline
\end{tabular}

distance-to-agreement since the effect of the Bragg peak degradation leads to a broadening of the Bragg peak and hence a small shift in the dose (compare Figs. 5 and 6). The allowed dose difference was set to $3 \%$ since this is roughly the average effect on the mean dose in the CTV for a modulation power of $800 \mu \mathrm{m}$. For a modulation power of $800 \mu \mathrm{m}$, the minimal passing rate is $90.4 \%$ for patient 5 and the beam direction $315^{\circ}$ corresponding to the maximum difference in the mean dose (compare Table 3). The average passing rate is $96.8 \%$. For a modulation power of $450 \mu \mathrm{m}$ the minimum passing rate is $93.1 \%$ and the average passing rate is $98.5 \%$. We also investigated

Table 5 Passing rates in percent of the gamma index 3\%/1 mm including all voxels with at least $20 \%$ of the maximum dose for all patients depending on the modulation power and beam direction

\begin{tabular}{lllll}
\hline Patient & $\begin{array}{l}\text { Modulation } \\
\text { power }\end{array}$ & \multicolumn{3}{l}{ Beam direction } \\
\cline { 3 - 5 } & \multicolumn{2}{l}{$0^{\circ}$} & $270^{\circ}$ & $315^{\circ}$ \\
\hline 1 & $450 \mu \mathrm{m}$ & 98.5 & 97.2 & 99.0 \\
& $800 \mu \mathrm{m}$ & 96.4 & 95.4 & 97.3 \\
& $450 \mu \mathrm{m}$ & 97.3 & 99.3 & 99.9 \\
3 & $800 \mu \mathrm{m}$ & 94.7 & 95.5 & 99.2 \\
& $450 \mu \mathrm{m}$ & 99.5 & 99.8 & 99.6 \\
4 & $800 \mu \mathrm{m}$ & 98.8 & 99.0 & 99.0 \\
& $450 \mu \mathrm{m}$ & 99.9 & 100 & 99.9 \\
5 & $800 \mu \mathrm{m}$ & 99.6 & 100 & 99.9 \\
& $450 \mu \mathrm{m}$ & 96.0 & 98.2 & 93.1 \\
& $800 \mu \mathrm{m}$ & 91.7 & 95.6 & 90.4 \\
\hline
\end{tabular}

the gamma index when including only those voxels with at least $80 \%$ of the maximum dose. For this gamma index the minimum passing rate is $84.0 \%$ with an average passing rate of $94.6 \%$ for a modulation power of $800 \mu \mathrm{m}$.

In order to assess whether the results from this study being derived using simple treatment plans can be used to estimate the dose uncertainty due to the Bragg peak degradation on more complex plans like IMPT plans, we investigated two IMPT plans - one each for patient 1 and 5. The reduction of the mean dose $D_{\text {mean }}$ of the CTV was $-1 \%$ for patient 1 and a modulation power of $450 \mu \mathrm{m}$. For patient 5 it was about $-3 \%$. For patient 1 , the reduction of the mean dose of the CTV approximately corresponds to the average dose reduction for the simple treatments plans with beam directions $0^{\circ}$, $270^{\circ}$ and $315^{\circ}$ (compare Table 3). For patient 5 the dose reduction for the IMPT plan is in the order of the maximum effect for the simple treatment plans.

\section{Discussion}

The influence of the Bragg peak degradation due to lung tissue on treatment plans of lung cancer patients was investigated. For all cases the treatment-planning system overestimated the dose delivered to the CTV and in some cases underestimated the dose delivered to distal OARs. This effect increases with an increasing modulation power. The maximum underestimation of the mean dose $D_{\text {mean }}$ is $-5 \%$ for the CTV and an extreme modulation power of $800 \mu \mathrm{m}$. The average underestimation is in the order $-2 \%$. This extreme modulation power of 
$800 \mu \mathrm{m}$ can occur in cases where a larger bronchial structure in the lung is positioned in the proton beam. However, for a more realistic modulation power of $450 \mu \mathrm{m}$, the underestimation of the mean dose $D_{\text {mean }}$ is only about $-3 \%$ at maximum. The average underestimation is roughly $-1 \%$.

Concerning the effects on OARs, it was shown that the effects are dependent on the beam direction which defines the relative position between the target volume and OAR for a given anatomy: As shown in Fig. 5 on the right side, the Bragg peak degradation results in a higher dose distal to the Bragg peak. Hence, only OARs distal to the PTV can receive a higher dose than predicted by the treatment-planning system. Due to range uncertainties in proton therapy it would typically be avoided to arrange fields in a way that an OAR is located directly distal to the PTV. Nevertheless, in some cases this is inevitable for example when the patient has been previously irradiated in this region or due to technical limitations of the beam delivery system. Additionally, anatomical characteristics could enforce an irradiation where an OAR is positioned distal to the PTV as it is the case with patient 3 as shown in Fig. 2: for the beam directions $315^{\circ}$ and $270^{\circ}$ the spinal cord is positioned distal to the PTV. However, for the beam direction $0^{\circ}$ the beam crosses the heart and the distance in lung is quite large. Since a patient's anatomy can oblige to use beams where an OAR is positioned distal to the PTV, we also investigated these cases. The underestimation of the mean dose $D_{\text {mean }}$ in the OARs trachea and spinal cord was $0.3 \mathrm{~Gy}$ at maximum. For the $\mathrm{D}_{2 \%}$ quantifying the maximum dose deposited in these OARs it was $1.5 \mathrm{~Gy}$ at maximum. The resulting enhanced dose deposited to OARs is far from any dose constraints used in the conventional treatment planning. Thus, the effects of the Bragg Peak degradation on OARs distal to the PTV are negligible for the cases investigated. However, in cases where the OAR is located directly distal to the PTV the effects might be larger and significant.

We were able to reproduce the findings from Flatten et al. [31] that the effects of the Bragg peak degradation increase with an increasing depth of the tumor in the lung and a decreasing tumor volume: for example, for patient 1 the underdosage of the CTV increases from $-0.5 \%$ to - $1 \%$ (for a modulation power of $450 \mu \mathrm{m}$ ) between the beam direction $270^{\circ}$ where the tumor depth is $3.3 \mathrm{~cm}$ and the beam direction $0^{\circ}$ where the depth is $4.6 \mathrm{~cm}$.

When comparing the results from patient 1 for the beam direction $315^{\circ}$ with the results from patient 5 for the beam direction $315^{\circ}$ one can see that in both cases the tumor is at a depth of $3.6 \mathrm{~cm}$ (see Table 1). However, the CTV of patient 1 is with $46.4 \mathrm{~cm}^{3}$ much larger compared to patient 5 with a volume of $2.7 \mathrm{~cm}^{3}$. The effect of the Bragg peak degradation on the mean dose in the CTV for patient 1 is with $-1 \%$ much smaller compared to patient 5 with $-3 \%$ (for a modulation power of $450 \mu \mathrm{m})$.

Regarding the number of fields used to irradiate the CTV, it was shown that as expected, the effect of the Bragg peak degradation is independent on the number of fields as long as these fields are optimized individually.

Concerning the complexity of the irradiation plans, we decided to investigate simple plans with only one single field as described in the Methods \& materials section. By investigating different beam directions, a large variety of scenarios (depth of tumor in lung, OAR distal to the PTV) has been covered and even for the worst cases the underdosage of the CTV was $-5 \%$ at maximum for an extreme modulation power of $800 \mu \mathrm{m}$ and only about $-3 \%$ for a more realistic modulation power of $450 \mu \mathrm{m}$. To assess whether these results can be applied to more complex treatment plans, we investigated two IMPT plans for a realistic modulation power of $450 \mu \mathrm{m}$. For both patients the reduction of the mean dose of the CTV was in the same order compared to the simple treatment plans. This supports the statement that the results found in this study - although being derived using simple treatment plans - can be used to estimate the dose uncertainties due to the Bragg peak degradation for more complex plans.

The passing rate of the gamma index was on average $96.8 \%$ for a modulation power of $800 \mu \mathrm{m}$ and $98.5 \%$ for a modulation power of $450 \mu \mathrm{m}$. The minimum passing rate for a realistic modulation power of $450 \mu \mathrm{m}$ was $93.1 \%$. The high passing rate of the gamma index is reasonable because as shown in Figs. 5 and 6 the Bragg peak degradation leads to a shift of the isodose lines. This shift is on average very small and hence covered by the distance-to-agreement in the gamma index. For all cases investigated in this study the passing rate was clinically acceptable. In addition to the finding that the reduction of the mean dose is on average only in the order of $-1 \%$ and at maximum $-3 \%$ for a realistic modulation power of $450 \mu \mathrm{m}$, this supports that the effects of the Bragg peak degradation are clinically tolerable.

What is more, it is well-known in the literature that the relative biological effectiveness (RBE) of protons is larger than 1.1 at the distal part of the Bragg peak [43]. At the moment, this change in RBE is not considered in commonly used treatmentplanning systems, hence, this larger biological effect might partially balance out the physical underdosage of the target volume due to the Bragg peak degradation which mainly occurs at the distal end of the Bragg peak and hence the target volume (compare Fig. 5). However, this change in RBE could also 
potentially increase the effects of the larger dose deposited in normal tissue distal to the Bragg peak.

The Bragg peak degradation due to lung tissue is only one of various issues in proton therapy in general and in proton therapy of lung cancer patients in particular as mentioned in the introduction. Thus, the dose and range uncertainties due to this degradation shall be compared to these other uncertainties in order to quantify its importance in the current clinical context. In a study by Paganetti [7] an overview of range uncertainties is given. Range uncertainties in proton therapy arise - among other things - from measurement uncertainties in water for commissioning $( \pm 0.3 \mathrm{~mm})$, patient setup $( \pm 0.7 \mathrm{~mm})$ or differences in the dose calculation between the treatment-planning system and Monte Carlo codes as a gold standard for dose calculation $( \pm 2 \mathrm{~mm})$. Other reasons for range uncertainties are due to the conversion of $x$-ray HU to stopping powers $( \pm 1 \%$ of the range) or biological effects $(\sim 0.8 \%$ of the range or $\sim 3 \mathrm{~mm}[14])$. These range uncertainties refer to the range of the $80 \%$ distal dose and correspond to average values. Furthermore, these uncertainties might be bigger in lung treatments [7]. The maximum range uncertainties for the $80 \%$ isodose lines due to the Bragg peak degradation based on an extreme modulation power of $800 \mu \mathrm{m}$ found in this study (see Table 2) are $10 \mathrm{~mm}$ in lung and $4 \mathrm{~mm}$ in tissue and hence are in the order of the mentioned average range uncertainties. Note that the values given by Paganetti [7] are average values while the range uncertainties investigated in this study are maximum values.

Additionally, range and dose uncertainties arise when changes of the anatomy due to weight loss or a shrinkage of the tumor are not accounted for. Szeto et al. [10] analyzed robust intensity modulated treatment plans of 16 patients with locally advanced NSCLC. The treatment dose was recalculated based on daily anatomy variations. Eight patients had an undercoverage of the target volume larger than $2 \mathrm{GyE}$ with a maximum of $12 \mathrm{GyE}$ in terms of the $\mathrm{D}_{99}$ (dose that is received by $99 \%$ of the target volume). With a prescribed dose of $66 \mathrm{GyE}$ this corresponds to relative deviations in the $\mathrm{D}_{99 \%}$ of $3 \%$ to $18 \%$. The maximum difference in the $\mathrm{D}_{98 \%}$ found in this study was $3 \%$ for a realistic modulation power of $450 \mu \mathrm{m}$.

Another crucial issue in proton therapy of lung cancer patients is motion. Dowdell et al. [20] investigated treatment plans for 5 lung cancer patients. Due to the interplay effects caused by the patient's motion, the mean dose in the target volume was only $88 \%$ to $92 \%$ of the prescribed dose. These interplay effects are however highly patient specific.

At last, we want to introduce and discuss two possible PTV concepts to account for and to avoid an underdosage of the target volume due to the Bragg peak degradation: following the range shifts as shown in Fig. 6, one possible PTV concept could be to increase the margin around the CTV at both the distal and proximal end. The effects of such a PTV concept are depicted in Fig. 7: the dose distributions in a water phantom downstream from $80 \mathrm{~mm}$ of lung tissue with a modulation power of $450 \mu \mathrm{m}$ are shown for the non-modulated (black) and the modulated case (yellow). The CTV marked with dashed lines is at depths between $34 \mathrm{~mm}$ and $47 \mathrm{~mm}$. In (a) the dose distributions can be seen for a PTV concept as used in this study with an isotropic margin of $3 \mathrm{~mm}$ around the CTV. The red line marks the prescribed dose within the CTV and PTV. In (b) the dose distributions are shown for the case where the PTV is the CTV plus a margin of $5 \mathrm{~mm}$ at the proximal end and a margin of $7 \mathrm{~mm}$ at the distal end. The dose coverage within the CTV and PTV is better compared to (a). The disadvantage of such a PTV concept is that the dose distribution reaches farther and hence leads to a higher integral dose in the normal tissue and maybe OARs distal to the PTV.

To avoid this larger range and additional dose deposited in normal tissue, another PTV concept might be used as depicted in Fig. 7c: in this case the same PTV concept as in (a) is used (isotropic margin of 3 $\mathrm{mm}$ around the CTV), however, during the treatment-planning process a larger dose is prescribed within the margin at both the proximal as well as the distal end. In this case we used a 3\% larger dose in the proximal and a $6 \%$ larger dose in the distal margin. By doing so a comparable dose distribution in the CTV as in Fig. 7b can be achieved, however, the dose deposited in normal tissue is smaller due to the shorter range. Note that such a PTV concept is connected to challenges since it is hard to guarantee a dose homogeneity in such a small volume. Furthermore, a difference of only $3 \%$ in dose in such a small volume is in the order of the uncertainties in proton therapy as discussed above, hence, it would hardly be possible to measure this difference in dose (e.g. as part of quality assurance).

For both PTV concepts an exact knowledge of the anatomy (depth of tumor in lung, tumor volume, location of tumor relative to soft tissue) is important to choose the appropriate values for the additional margin (case b) or the additional dose (case c). Furthermore, the knowledge of the modulation power within the lung tissue is important, since this defines the range and dose uncertainties connected to the Bragg peak degradation. Unfortunately, there is currently no solution to determine the modulation power in patients in-vivo. This is a critical issue still to be solved.

Altogether, the effects of the Bragg peak degradation are at maximum about $5 \%$ concerning the 

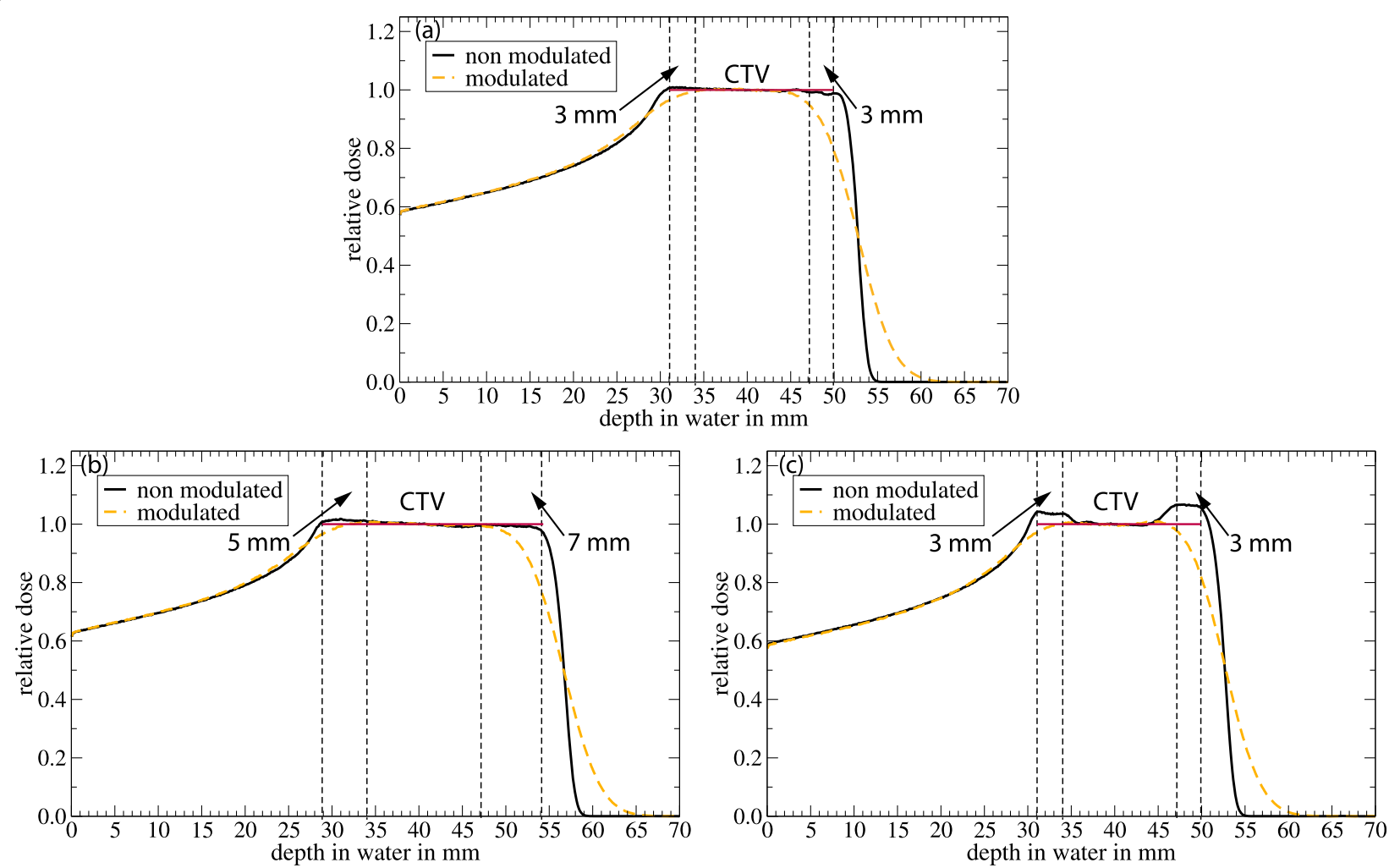

Fig. 7 The effects of different PTV concepts. In a the effects for a PTV as used in this study consisting of an isotropic margin of $3 \mathrm{~mm}$ around the CTV are shown. In $\mathbf{b}$ for a PTV concept that has a $5 \mathrm{~mm}$ margin at the proximal end and a $7 \mathrm{~mm}$ margin at the distal end of the CTV. In $\mathbf{c}$ for the same PTV concept as used in a, however, in this case the prescribed dose within this margin is larger than the prescribed dose in the CTV. In black the dose distribution for the non-modulated case and in yellow the dose distribution for the modulated case based on a modulation power of $450 \mu \mathrm{m}$. The CTV and the margin as well as the resulting PTV are marked in dashed lines. The red line marks the prescribed dose within the PTV

underestimation of the mean dose $\mathrm{D}_{\text {mean }}$ in the CTV when optimizing the treatment plan without considering the degradation due to the lung tissue. Compared to the range and dose uncertainties in proton therapy of lung cancer patients due to the addressed reasons, the effects of the Bragg peak degradation are clinically tolerable to a certain degree in the current clinical context. However, these mentioned dose uncertainties are constantly being reduced which might change this clinical context. Hence, a consideration of the Bragg peak degradation could become more relevant in the future and would bring proton therapy for lung cancer patients closer to a high-precision therapy. The effects of the degradation might be accounted for in the treatment-planning process by applying a corresponding PTV concept as suggested in this study. What is more, this PTV concept and hence the dose deposition in the patient could be optimized when having a detailed knowledge of the lung tissue's modulation power. In our opinion, the exact determination of this modulation power is one crucial issue still to be solved.

\section{Conclusion}

The effects of the Bragg peak degradation due to lung tissue on lung cancer patients were investigated. The maximum effect on the mean dose $\mathrm{D}_{\text {mean }}$ in the CTV according to this study was about $5 \%$ at maximum for an extreme modulation power of $800 \mu \mathrm{m}$, a long distance travelled through lung and a small tumor volume. For a more realistic modulation power of $450 \mu \mathrm{m}$ the maximum effect was only about $3 \%$ in terms of $D_{\text {mean }}$. For OARs the effect was negligible for the cases investigated. This study confirms that the effects of the Bragg peak degradation are clinically tolerable to a certain degree in the current clinical context considering the various more critical dose uncertainties due to motion and range uncertainties in proton therapy. Furthermore, these effects might be accounted for by using corresponding PTV concepts as suggested in this study.

\section{Abbreviations}

CTV: Clinical target volume; $D_{20}$ : Dose that is received by $2 \%$ of a structure's volume; $D_{98 \%}$ : Dose that is received by $98 \%$ of a structure's volume;

$D_{\text {mean: }}$ Average dose within a structure; IMPT: Intensity modulated proton 
therapy; OAR: Organ at risk; PTV: Planning target volume; RBE: Relative biological effectiveness; SBRT: Stereotactic body radiation therapy

\section{Acknowledgements}

We would like to thank the reviewers which helped to improve this manuscript.

\section{Authors' contributions}

K-SB used the mathematical model to generate the density modulation functions needed for the density modulation to reproduce the Bragg peak degradation in the Monte Carlo simulations. K-SB created the densitymodulated DICOM sets and executed the Monte Carlo simulations as well as analyzed the DVHs produced with Eclipse. K-SB wrote the manuscript. VF optimized the treatment plans and transferred these optimized plans to the Monte Carlo code TOPAS and subsequently generated the DVHs. UW contributed to the mathematical model to consider the Bragg peak degradation in Monte Carlo codes underlying to this study. SL and FE contributed to this work by contouring the DICOM sets and helped with clinical information on treatment plans. KZ substantively revised this work and was supervising the work. RE-C substantively revised this work and was supervising the work.

\section{Funding}

We did not receive any funding for this work.

\section{Availability of data and materials}

The datasets generated during and/or analyzed during the current study are available from the corresponding author on reasonable request.

\section{Ethics approval and consent to participate}

Not applicable.

\section{Consent for publication}

Not applicable.

\section{Competing interests}

The authors declare that they have no competing interests.

\section{Author details}

${ }^{1}$ University Medical Center Giessen-Marburg, Department of Radiotherapy and Radiooncology, Marburg, Germany. ${ }^{2}$ University of Applied Sciences, Institute of Medical Physics and Radiation Protection, Giessen, Germany. ${ }^{3} \mathrm{GSI}$ Helmholtzzentrum für Schwerionenforschung, Biophysics Division, Darmstadt Germany. ${ }^{4}$ Marburg Ion-Beam Therapy Center (MIT), Marburg, Germany.

${ }^{5}$ Frankfurt Institute of Advanced Studies - FIAS, Frankfurt, Germany.

Received: 19 February 2019 Accepted: 6 September 2019

Published online: 25 October 2019

\section{References}

1. Wilson RR. Radiological use of fast protons. Radiology. 1946;47:487-91.

2. Smith AR. Proton therapy. Phys Med Biol. 2006;51(13):R491.

3. Chang JY, Zhang $X$, Wang $X$, et al. Significant reduction of normal tissue dose by proton radiotherapy compared with three-dimensional conformal or intensity-modulated radiation therapy in stage I or stage III non-smallcell lung cancer. Int J Radiat Oncol Biol Phys. 2006;65(4):1087-96.

4. Chang JY, Jabbour SK, de RD, et al. Consensus statement on proton therapy in early-stage and locally advanced non-small cell lung cancer. Int J Radiat Oncol Biol Phys. 2016:95(1):505-16.

5. Nichols RC, Huh SN, Henderson RH, et al. Proton radiation therapy offers reduced normal lung and bone marrow exposure for patients receiving dose-escalated radiation therapy for unresectable stage iii non-small-cell lung cancer: a dosimetric study. Clin Lung Cancer. 2011;12(4):252-7.

6. Chang JY, Komaki R, Lu C, et al. Phase 2 study of high-dose proton therapy with concurrent chemotherapy for unresectable stage III nonsmall cell lung cancer. Cancer. 2011;117(20):4707-13.

7. Paganetti $\mathrm{H}$. Range uncertainties in proton therapy and the role of Monte Carlo simulations. Phys Med Biol. 2012;57(11):R99-117.

8. Unkelbach J, Chan TCY, Bortfeld T. Accounting for range uncertainties in the optimization of intensity modulated proton therapy. Phys Med Biol. 2007; 52(10):2755-73.
9. Hui Z, Zhang X, Starkschall G, et al. Effects of interfractional motion and anatomic changes on proton therapy dose distribution in lung cancer. Int J Radiat Oncol Biol Phys. 2008;72(5):1385-95.

10. Szeto $Y Z$, Witte $M G$, van Kranen SR, et al. Effects of anatomical changes on pencil beam scanning proton plans in locally advanced NSCLC patients. Radiother Oncol J Eur Soc Ther Radiol Oncol. 2016;120(2):286-92.

11. Hoffmann $L$, Alber M, Jensen MF, et al. Adaptation is mandatory for intensity modulated proton therapy of advanced lung cancer to ensure target coverage. Radiother Oncol J Eur Soc Ther Radiol Oncol. 2017;122(3):400-5.

12. España $\mathrm{S}$, Paganetti $H$. The impact of uncertainties in the $C T$ conversion algorithm when predicting proton beam ranges in patients from dose and PET-activity distributions. Phys Med Biol. 2010:55(24):7557-71.

13. Arbor N, Dauvergne D, Dedes G, et al. Monte Carlo comparison of x-ray and proton $\mathrm{CT}$ for range calculations of proton therapy beams. Phys Med Biol. 2015;60(19):7585-99.

14. Carabe A, Moteabbed M, Depauw N, et al. Range uncertainty in proton therapy due to variable biological effectiveness. Phys Med Biol. 2012;57(5): 1159-72.

15. Bert C, Grözinger SV, Rietzel E. Quantification of interplay effects of scanned particle beams and moving targets. Phys Med Biol. 2008:53:2253-65.

16. Bert C, Durante M. Motion in radiotherapy: particle therapy. Phys Med Biol. 2011;56:R113.

17. Grassberger C, Dowdell S, Lomax A, et al. Motion interplay as a function of patient parameters and spot size in spot scanning proton therapy for lung cancer. Int J Radiat Oncol Biol Phys. 2013;86(2):380-6.

18. Moyers MF, Miller DW, Bush DA, et al. Methodologies and tools for proton beam design for lung tumors. Int J Radiat Oncol Biol Phys. 2001:49(5):1429-38.

19. Bert C, Saito N, Schmidt A, et al. Target motion tracking with a scanned particle beam. Med Phys. 2007:34(12):4768-71.

20. Dowdell S, Grassberger C, Sharp GC, et al. Interplay effects in proton scanning for lung: a 4D Monte Carlo study assessing the impact of tumor and beam delivery parameters. Phys Med Biol. 2013;58(12):4137-56.

21. Urie $M$, Goitein $M$, Holley WR, et al. Degradation of the Bragg peak due to inhomogeneities. Phys Med Biol. 1986;31:1.

22. Sawakuchi GO, Titt U, Mirkovic D, et al. Density heterogeneities and the influence of multiple coulomb and nuclear scatterings on the Bragg peak distal edge of proton therapy beams. Phys Med Biol. 2008:53(17):4605-19.

23. Goitein M. The measurement of tissue heterodensity to guide charged particle radiotherapy. Int J Radiat Oncol Biol Phys. 1977;3:27-33.

24. Perles LA, Mirkovic D, Sawakuchi GO, et al. Monte Carlo investigation of rebinning material density distributions of lung parenchyma phantoms in proton therapy. Nucl Technol. 2011;175(1):22-6.

25. Sell M, Titt U, Perles $L$, et al. WE-E-BRB-02: evaluation of analytical proton dose predictions with a lung-like plastic phantom. Med Phys. 2012;39(6):3956

26. Titt U, Sell M, Unkelbach J, et al. Degradation of proton depth dose distribution attributable to microstructures in lung-equivalent material. Med Phys. 2015;42(11):6425

27. Ringbaek TP, Simeonov $Y$, Witt M, et al. Modulation power of porous materials and usage as ripple filter in particle therapy. Phys Med Biol. 2017; 62:2892.

28. Witt M, Weber $U$, Simeonov $Y$, et al. SU-E-T-671: range-modulation effects of carbon ion beams in lung tissue. Med Phys. 2015:42(6):3491.

29. Baumann K-S, Witt W, Weber U, et al. An efficient method to predict and include Bragg curve degradation due to lung-equivalent materials in Monte Carlo codes by applying a density modulation. Phys Med Biol. 2017, 62: 3997-4016.

30. Espana S, Paganetti H. Uncertainties in planned dose due to the limited voxel size of the planning $C T$ when treating lung tumors with proton therapy. Phys Med Biol. 2011;56(13):3843.

31. Flatten V, Baumann K-S, Weber U, et al. Quantification of the dependencies of the Bragg peak degradation due to lung tissue in proton therapy on a CT-based lung tumor phantom. Phys Med Biol. 2019;64:155005.

32. Hata M, Tokuuye $K$, Kagei $K$, et al. Hypofractionated high-dose proton beam therapy for stage I non-small-cell lung cancer: preliminary results of a phase I/II clinical study. Int J Radiat Oncol Biol Phys. 2007:68(3):786-93.

33. Bush DA, Slater JD, Shin BB, et al. Hypofractionated proton beam radiotherapy for stage I lung cancer. Chest. 2004:126(4):1198-203.

34. Chen J, Lu JJ, Ma N, et al. Early stage non-small cell lung cancer treated with pencil beam scanning particle therapy: retrospective analysis of early results on safety and efficacy. Radiat Oncol (London, England). 2019;14(1):16. 
35. Góra J, Stock M, Lütgendorf-Caucig C, et al. Is there an advantage in designing adapted, patient-specific PTV margins in intensity modulated proton beam therapy for prostate cancer? Int I Radiat Oncol Biol Phys. 2013:85(3):881-8.

36. Park PC, Zhu XR, Lee AK, et al. A beam-specific planning target volume (PTV) design for proton therapy to account for setup and range uncertainties. Int J Radiat Oncol Biol Phys. 2012;82(2):e329-36.

37. Perl J, Shin J, Schuemann J, et al. TOPAS: an innovative proton Monte Carlo platform for research and clinical applications. Med Phys. 2012;39(11):6818-37.

38. Agostinelli S, et al. Geant4 - a simulation toolkit. Nucl Instrum Methods Phys Res A. 2003;506:250-303.

39. Taylor PA, Kry SF, Followill DS. Pencil beam algorithms are unsuitable for proton dose calculations in lung. Int J Radiat Oncol Biol Phys. 2017;99(3):750-6.

40. Witt M. Modulationseffekte von Kohlenstoffionen bei der Bestrahlung von Lungen, master thesis, University of Applied Sciences, Giessen, Germany. URL: https://www.thm.de//se/images/user/KZink-105/Abschlussarbeiten/ Masterarbeit_Matthias_Witt_2014.pdf (last called: 11/09/2019).

41. International Commission on Radiation Units and Measurements (ICRU). ICRU report volume 50: prescribing, recording, and reporting photon beam therapy. 1993.

42. International Commission on Radiation Units and Measurements (ICRU). ICRU report volume 62: prescribing, recording and reporting photon beam therapy (supplement to ICRU report 50). 1999.

43. Paganetti H, Blakely E, Carabe-Fernandez A, et al. Report of the AAPM TG256 on the relative biological effectiveness of proton beams in radiation therapy. Med Phys. 2019;46(3):e53-78.

\section{Publisher's Note}

Springer Nature remains neutral with regard to jurisdictional claims in published maps and institutional affiliations.

Ready to submit your research? Choose BMC and benefit from:

- fast, convenient online submission

- thorough peer review by experienced researchers in your field

- rapid publication on acceptance

- support for research data, including large and complex data types

- gold Open Access which fosters wider collaboration and increased citations

- maximum visibility for your research: over $100 \mathrm{M}$ website views per year

At BMC, research is always in progress.

Learn more biomedcentral.com/submissions 